\title{
Harold Gramatges, análisis musicográfico del Móvil II
}

\author{
Harold Gramatges, musicographic analysis of Móvil II
}

\author{
Rafael Guzmán Barrios \\ Universidad de las Artes de Ecuador \\ rafael.guzman@uartes.edu.ec \\ ORCID iD: https://orcid.org/0000-0002-1466-4209
}

\section{RESUMEN}

El análisis de la vanguardia musical cubana de los años 60 es todavía una tarea incompleta de la Musicología. La presente investigación tiene como objetivo principal realizar un análisis integral (espacio, tiempo, instrumentación, dinámicas, dramaturgia) de la obra Móvil II del compositor cubano Harold Gramatges (Santiago de Cuba, 1918 - La Habana, 2008), a través de la partitura y su registro sonoro. Esta pieza es la segunda de su serie Móviles (1969-80), y para estudiarla se crearon gráficos que visualizan los medios expresivos y las técnicas empleadas por el compositor, y ayudaron a valorar holísticamente los múltiples elementos que conforman el discurso musical. Además, el trabajo propone este método musicográfico, como una herramienta metodológica más para el análisis musical, y contribuye a una mayor visualización de la obra del compositor cubano. Los gráficos diseñados para la presente investigación no fueron influenciados por la visualidad de las partituras del compositor, o sus comentarios durante las entrevistas realizadas.

Palabras clave: Gramatges, gráficos, vanguardia, análisis, Móviles.

\section{ABSTRACT}

The analysis of the Cuban musical avant-garde of the 1960s is still an incomplete task of Musicology. The main objective of this research is to carry out a comprehensive analysis (space, time, instrumentation, dynamics, dramaturgy) of the mobile work II by the Cuban composer Harold Gramatges (Santiago de Cuba, 1918 - Havana, 2008), through the score and its sound record. This piece is the second in his series Mobiles (1969-80), and to study 


\section{RAFAEL GUZMÁN BARRIOS}

it, graphics were created that visualize the expressive means and techniques used by the composer, and helped to holistically assess the multiple elements that make up the musical discourse. In addition, the work proposes this musicographic method as one more methodological tool for musical analysis and contributes to a greater visualization of the Cuban composer's work. The graphics designed for the present investigation were not influenced by the visuality of the composer's scores, or his comments during the interviews.

Key Words: Gramatges, graphics, vanguard, analysis, Móviles.

Guzmán Barrios, R. (2021). Harold Gramatges, análisis musicográfico del Móvil II. Cuadernos de Investigación Musical, (12), pp. 79-101.

\section{INTRODUCCIÓN}

El Móvil II del compositor cubano Harold Gramatges, pertenece a la serie Móviles (1969-80), y fue creado durante la etapa de mayor riqueza creativa (en cuanto a variedad de formatos y cantidad de obras compuestas) en la producción cameral del creador. Esta obra es la más osada de la serie debido a su concepción temporal, tímbrica y espacial. Siete intérpretes bajo la batuta de un director asumirán cinco instrumentos y dos grupos de abundante percusión; estos además estarán distribuidos espacialmente en la escena según el diseño preestablecido. La obra fue dedicada al director de orquesta Manuel Duchezne Cuzán (La Habana, 1932 - 2005) y el Conjunto Instrumental Nuestro Tiempo, y estrenada en la Sala Hubert de Blanck de la Habana, el 27 de septiembre de 1972, durante el Encuentro de Música Latinoamericana de la Casa de las Américas (Acosta, 2001).

\section{ANTECEDENTES}

Se han encontrado numerosos materiales (entrevistas, videos, entre otros) donde se prioriza al Harold Gramatges pensador, pedagogo y humanista. La mayor parte de la obra del compositor se encuentra grabada y publicada, sin embargo, solo una pequeña parte de su catálogo ha sido objeto de estudio y análisis más allá de lo hallado en notas discográficas, prólogos y algunos artículos. En ese sentido se han encontrado:

- La tesis de grado y doctorado Eclecticism in modern cuban music as reflected in selected piano works by Harold Gramatges: an investigative analysis de Milvia Rodríguez (University of Nebraska, 2006). En este documento la autora analiza tres obras para piano del compositor, pertenecientes a tres periodos estéticamente diferentes en la carrera de Gramatges. 


\section{HAROLd GRAMATGES, ANÁLISIS MUSICOGRÁFICO DEL MóVIL II}

Móvil I is Harold Gramatges's pianistic journey into the avant-garde movement. The piece reflects experimental ideas concerning space and sound, as the composer expands the role of the performer by combining improvisation and written-out motivic elements that expand and develop throughout the piece (Rodríguez, 2006, p. 1).

- Las tesis de grado y doctorado Aproximaciones al problema de lo nacional y lo internacional en la obra de los compositores cubanos y Trayecto de lo cubano en un espacio de tiempo respectivamente, ambas de la musicóloga Marta Rodríguez Cuervo. En el primero de estos trabajos la autora realiza el análisis musicológico de la obra Serenata (1947) de Harold Gramatges.

- Artículos sobre el compositor y sus obras como son: Móviles de Gramatges de Mayra A. Martínez en Revolución y Cultura No 9. Septiembre, 1987; Harold Gramatges Móvil I ¿azar? de Aimée Guerrero en revista de música Clave No 1, La Habana 2002; Tres Preludios de Harold Gramatges I, II y III de Miriam Pérez Fleitas en las revistas Clave $\mathrm{N}^{\circ}$ 7, 8 y 9. 1987-88, entre otros. En este último la musicóloga efectúa, en tres partes, el análisis musical de la obra Tres preludios a modo de Tocata (1952-1953).

- Diccionarios y libros como Presencia de la Revolución en la música cubana (1983) del propio compositor y el prólogo Los Móviles de Harold Gramatges de Leonardo Acosta para la edición Facsimilar Móviles (2001) de la Editorial Letras Cubanas.

En la bibliografía examinada el Móvil II no ha sido analizado profunda e integralmente. En el prólogo Los Móviles de Harold Gramatges, Leonardo Acosta solo esboza algunas de sus características esenciales como son: influencias de otras obras, cubanía y de manera general la morfología, la instrumentación, la Intertextualidad y los procedimientos técnicosmusicales.

Respecto al análisis músico-gráfico que se propone es necesario precisar:

- No es objetivo visibilizar geométricamente los sonidos y las vibraciones de la obra de Gramatges desde la Cimática, por lo que fuentes como Cimática: las formas e imágenes del sonido, una aproximación desde la experiencia personal de M. Arce (2011), Universidad del País Vasco/Euskal Herriko Unibertsitatea, no se adecúan al propósito de la investigación.

- En el trabajo Análisis gráfico-sonoro como fuente de conocimiento de la obra musical en estudiantes de la Universidad Internacional de La Rioja (UNIR) de M. Vela, (Universidad Internacional de La Rioja), se propone un análisis músico-gráfico sobre la partitura que se analiza, pero en la presente investigación los gráficos son independientes a la partitura, aunque reflejan el corpus musical y su registro sonoro.

-Tampoco se propone un método para el análisis de la música visual como Isaac Diego García-Fernández realiza en su Estrategias para el análisis de la música visual: el caso de Llorenç Barber (Universidad Internacional de La Rioja, 2019). Aunque tiene pinceladas de novedosa visualidad, el Móvil II mantiene la notación escrita tradicional. 


\title{
RAFAEL GUZMÁN BARRIOS
}

Los gráficos creados fueron realizados en Word Office, y no generados por software especialmente diseñados para el análisis de música electroacústica y la obtención de sonogramas, espectrogramas o envolventes dinámicas.

\section{Problema}

¿Qué elementos, provenientes de la vanguardia musical de los 60, incorpora Gramatges en su obra Móvil II? En ese sentido la presente investigación se propone como objetivo principal realizar un análisis integral (espacio, tiempo, instrumentación, dinámicas, dramaturgia) de la obra a través de la partitura y su registro sonoro.

\section{Metodología}

Se alternó el análisis de la obra con acercamientos generales y específicos, y a través de una red de relaciones que protagonizaron los medios expresivos (Padilla, 1999, p. 11). Para descubrir las leyes que rigen su engranaje, se han utilizado las categorías que propone el compositor Pierre Boulez (Montbrison, 1925 - Baden-Baden, 2016) sobre tiempo musical. En ese sentido, el francés cataloga el tiempo musical de dos maneras: liso y estriado.

\begin{abstract}
Dispongamos por debajo de una línea de referencia una superficie perfectamente lisa y una superficie estriada, regular o irregularmente, poco importa; desplacemos esta superficie lisa ideal, $\mathrm{n}$ podremos reparar ni en la velocidad ni en el sentido del desplazamiento, al no encontrar el ojo ningún punto de apoyo sobre el que apoyarse; con la superficie estriada, por el contrario, el desplazamiento se presentará tanto en su velocidad como en su sentido. El tiempo amorfo es comparable a la superficie lisa, el tiempo pulsado a la superficie estriada; por esta razón, por analogía, denominaría las dos categorías así definidas con el nombre de tiempo liso y tiempo estriado. (Boulez, 2009, pp. 132-133)
\end{abstract}

Por otro lado, también resultan muy útiles y efectivas las categorías que concibe en el campo de la dinámica:

Por dinámica-punto, entendemos todo grado fijo de la dinámica; los encadenamientos se harán de punto a punto sobre la escala elegida, sin que haya, de uno a otro recorrido, gesto. Con la dinámica-línea, por el contrario, operaremos sobre los trayectos de una amplitud dada a una nueva amplitud: crescendo-decrescendo, y sus combinaciones. Esta segunda categoría, la definiría como si proviniera del glissando dinámico, comparable al glissando de las alturas, y en los tempi (acelerando, ritardando) (Boulez, 2009, p. 93) 


\section{HAROLD GRAMATGES, ANÁLISIS MUSICOGRÁFICO DEL MóVIL II}

Con el objetivo de analizar el espacio musical se consideran dos tipos de nociones de espacio en la música expuestos por el musicólogo Rubén López Cano: espacialidad intramusical y externomusical (López Cano, 2001, pp.101-122). La espacialidad intramusical aborda los procesos de dosificación sonora en la textura. La materialización de estas en el interior de una obra puede tener su origen en la fuente sonora, o en las trasformaciones registradas en otras variables como la intensidad o la organización de alturas. La espacialidad externomusical apunta a la organización de los focos sonoros en el espacio donde se realiza el performance (Guzmán, 2019, p. 61).

Para facilitar el proceso analítico, se recurre al diseño de gráficos y tablas que auxiliaron a valorar tanto la totalidad como los detalles del corpus musical. A través de los gráficos se convirtió el discurso sonoro en representaciones visuales. Mediante esta propuesta metodológica, los estudiantes pueden obtener, simultáneamente, una visión general de la obra y otra fragmentada en distintas variables analíticas. De esta forma, cuentan con una herramienta que les permite apreciar con claridad las relaciones internas de la obra musical (Vela, 2018). Parámetros como la dramaturgia, el tiempo, el espacio, la intensidad y la instrumentación serán plasmados visualmente en un proceso inverso al realizado por el compositor, pues la serie Móviles está inspirada en las piezas homónimas del escultor Alexander Calder (Gramatges, 2001).

De acuerdo a José A. Sánchez en su ensayo Dramaturgia en el campo expandido:"...la dramaturgia está más allá o más acá del texto, se resuelve siempre en el encuentro inestable de los elementos que componen la experiencia escénica”. (Sánchez, 2010, p. 20). En el presente análisis se aborda una obra sin texto, pero se propone el análisis de la dramaturgia a través de los medios expresivos que participan en la puesta en marcha del discurso musical.

Se ha utilizado además el término gesto composicional. De acuerdo a Vinasco (2011) ellos serían:

Escritos no verbales: como los que encontramos en las partituras, haciendo la salvedad que no deberán ser confundidos con los códigos de que se componen, ya que un gesto es una unidad de mayores alcances, capaz de generar en el receptor múltiples significados... Auditivos: se trata de la realización sonora de un gesto, por lo general a partir de un gesto escrito en partitura, el cual es llevado a la práctica (p. 20).

Para este caso se acomoda al objetivo de investigación y serían recursos que utiliza el compositor y que ayudarán a singularizar su obra y su estilo de componer.

Para el análisis a nivel detallado se proponen dos términos: Estructuras y Desplazamientos. En su ensayo sobre las diferentes acepciones de Forma y Estructura, Santiago Pérez, Aldeguer concluye que: "Entendemos las diferencias de estructura musical generalmente como un micro dentro de un macro, compuesto por temas, frases, semifrases, etc. Mientras que la forma conlleva la macro: la suite, la sinfonía, la fuga, la canción, etc.” (Pérez, 2011, p. 97). 


\section{RAFAEL GUZMÁN BARRIOS}

Para el propósito de esta investigación también se analiza el micro, pero se precisa el término Estructuras a las diversas organizaciones que formen dos (simples) o más sonidos (compuestas) en la vertical del espacio musical. Podrán clasificarse de acuerdo a su distribución en el espacio intramusical en: simétricas, asimétricas, cerradas (clústeres), semicerradas (semiclústeres), abiertas (cuando existen distancias mayores a la octava entre los sonidos) e híbridas (clústeres separados por amplios intervalos). Además, pueden ser catalogadas de acuerdo a la organización de alturas o escalas utilizada en: cromáticas, diatónicas, tonos enteros, pentáfonas y mixtas. Los Desplazamientos serán secuencias de dos o más sonidos intercalados o no por silencios.

Como parte de la metodología cualitativa aplicada se utiliza la entrevista como recurso crucial de la investigación, pues era una oportunidad de lujo aprovechar la presencia física del compositor. Es preciso aclarar que los resultados de estas conversaciones registradas no influyeron en el proceso de la investigación, y el compositor validó como positivas las conclusiones alcanzadas respecto al análisis de su obra.

\section{INSTRUMENTACIÓN}

El mundo sonoro del Móvil II es una constante evocación a lugares y momentos inexplorados. La mezcla de instrumentos tradicionalmente usados (corno y flauta), con otros que hasta la primera mitad del siglo XX eran considerados marginales, anecdóticos y pintorescos (celesta, vibráfono, campanelli, xilófono) y la abundante presencia de percusión especialmente la cubana, provoca la intersección de disímiles ejes geográficos y cronológicos.

En el Móvil II pensé en la presencia de dos instrumentos solistas que son simbólicos dentro de la prehistoria de la música: el caracol que pudiera ser el origen del corno, y la flauta que un hueso pudo haber sido su ascendente más lejano. Estos dos instrumentos los rodeo de teclados (piano, vibráfono, xilófono) y un pequeño grupo de percusión cubana (H. Gramatges, comunicación personal, 21 de septiembre de 2005).

En otro momento de la entrevista recalca: "Los instrumentos como el piano, el vibráfono y la celesta constituyen el ropaje armónico de los dos protagonistas melódicos, la flauta y el corno". (H. Gramatges, comunicación personal, 21 de septiembre de 2005).

El compositor declara que la flauta y el corno son los instrumentos solistas o protagonistas en el Móvil II. Sin embargo, en el registro sonoro no se aprecia dicho protagonismo, ni se percibe a los instrumentos de percusión con afinación determinada como ropaje armónico solamente. Se considera que uno de los protagonistas de esta obra es el equilibrio instrumental y sus conflictos:

- Maderas - el resto del formato.

- Percusión de sonido determinado - de sonido indeterminado.

- Percusión I - percusión II. 


\section{HAROLD GRAMATGES, ANÁLISIS MUSICOGRÁFICO DEL MóVIL II}

Ellos son felizmente resueltos sin que ningún elemento haya resultado más destacado que el resto.

Por otro lado, Gramatges explora toda la tesitura de la flauta (evento I) incluyendo algunos efectos como son vibraciones microtonales (evento A) y frullatos (evento I). No se puede decir lo mismo sobre el corno el cual es explotado fundamentalmente en sus registros medio (eventos A, B...) y grave (eventos $\mathrm{D}$ y J), aunque en el evento $\mathrm{D}$ el instrumentista realiza efectos con la mano y el pabellón del instrumento. Vuelve el piano a ser una rica fuente de colores para el compositor cuando aprovecha toda la tesitura de su cordaje (empleando baquetas, yema de los dedos y uñas) y teclado, así como determinados efectos como el empleado en el evento E. En la percusión de sonido determinado tenemos la celesta que se desempeña en su registro medio-agudo (eventos C, J y L), el campanelli o glockenspiel que aprovecha toda su estrecha tesitura e incluso coquetea con el Bb que está por debajo del C central (eventos B y K) y el vibráfono y xilófono recrean sus respectivos espacios intramusicales totalmente (no incluimos los eventos y fragmentos donde el intérprete improvisa o determina la altura a ejecutar). Los sonidos de la celesta, el glockenspiel y el vibráfono resultan a la octava superior de lo que están escritos.

A continuación se muestra mediante tablas un análisis de las entradas y salidas de los instrumentos en cada uno de los eventos. En el eje horizontal se refleja el tiempo, y en el vertical la instrumentación de cada evento.

1) Flauta (Fl)

2) Corno (Cor)

3) Piano-celesta (P-Cel).

4) Vibráfono-Campanelli (Vbf-Camplli)

5) Xilófono (Xil)

6) Percusión I: Triángulo (Trg.)

3 Platos suspendidos. (pl.) Agudo, medio y grave (a, m, g)

3 Gongs. Agudo, medio y grave (a, $\mathrm{m}, \mathrm{g}$ )

Claves (clav.)

Maracas (marc.)

7) Percusión II: 2 Bongoes (bong) Agudo y grave (a,g)

2 Tambores (tumbadoras) (tumb) Agudo y grave (a,g)

3 Tom toms (ttos). Agudo, medio y grave (a, m, g)

Claves (clav.)

Maracas (marc.)

$\downarrow$ Entrada de instrumento

$\uparrow$ Salida de instrumento

......se continúa interpretando. 


\section{RAFAEL GUZMÁN BARRIOS}

Como se puede observar en la Tabla 1, en el Evento A los instrumentos que intervienen aparecen y desaparecen progresivamente, dosificando tímbricamente su paleta sonora en el comienzo de la obra; a diferencia del Evento B donde ya el compositor busca combinaciones tímbricas en sus ataques.

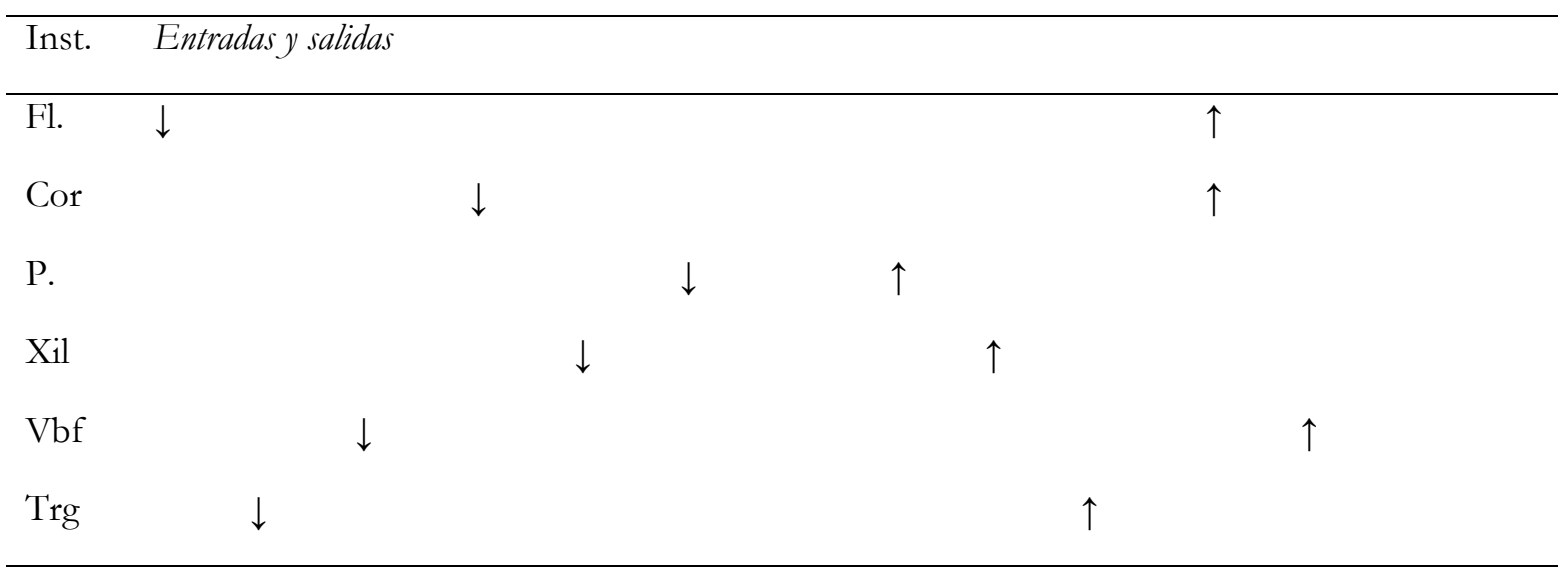

Tabla 1. Entradas y salidas de instrumentos Evento $A$.

Fuente: Elaboración propia a partir del análisis de la instrumentación de Móvil II (Gramatges, 2001)

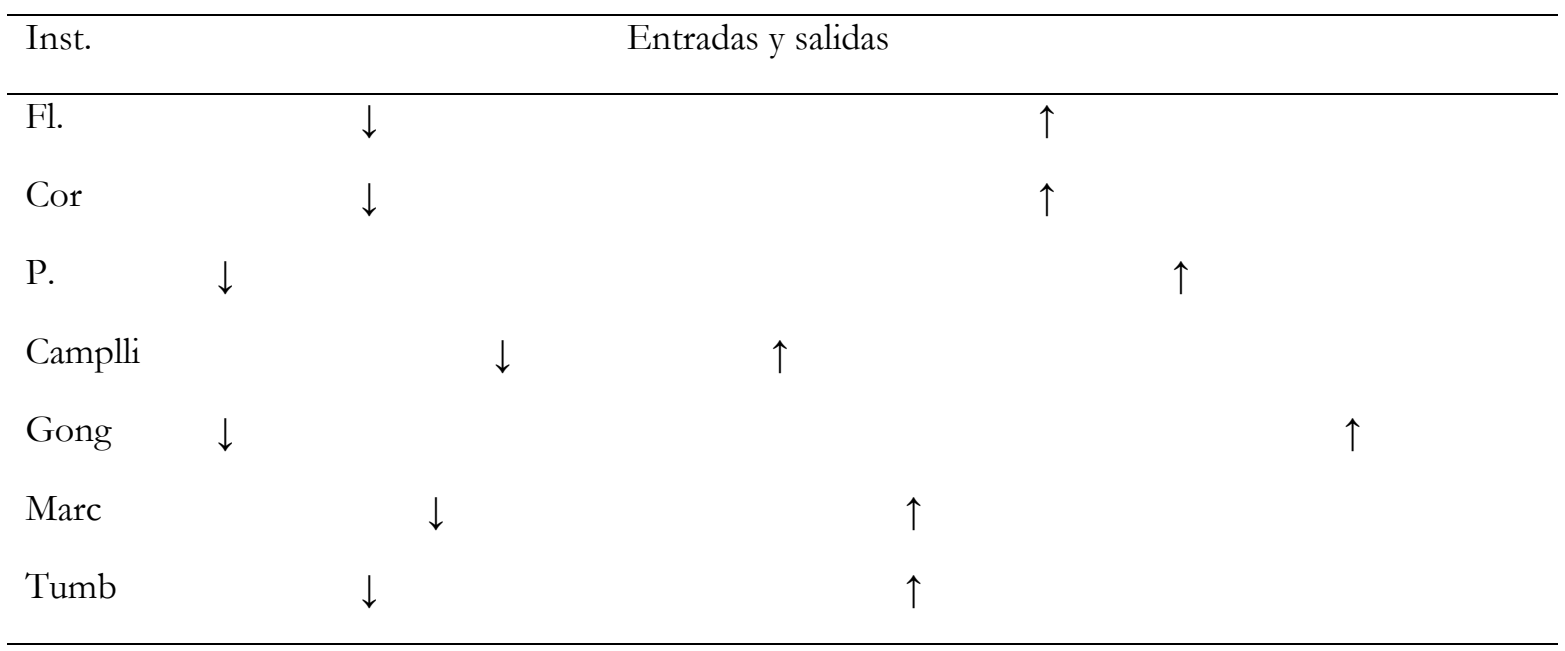

Tabla 2. Entradas y salidas de instrumentos Evento B.

Fuente: Elaboración propia a partir del análisis de la instrumentación de Móvil II (Gramatges, 2001)

La flauta y el corno se han mantenido como denominador común, y en el Evento C hubo una entrada simultánea de la mayoría de los instrumentos. 


\begin{tabular}{lcccc}
\hline Inst. & \multicolumn{4}{c}{ Entradas y salidas } \\
\hline Fl. & $\downarrow$ & $\uparrow$ & $\ldots \ldots \ldots \ldots$ \\
Cor & & $\downarrow$ & \\
Cel & $\downarrow$ & $\uparrow$ & \\
P & $\downarrow$ & $\uparrow$ & & \\
Vbf & $\downarrow$ & & $\uparrow$ & \\
Bong & $\downarrow$ & & & \\
Tumb & $\downarrow$ & & & \\
Ttos & $\downarrow$ & & & \\
& & & & \\
\end{tabular}

Tabla 3. Entradas y salidas de instrumentos Evento $C$.

Fuente: Elaboración propia a partir del análisis de la instrumentación de Móvil II (Gramatges, 2001).

\begin{tabular}{|c|c|c|c|c|}
\hline Inst. & & Entradas y salidas & & \\
\hline Cor & f............ & & $\uparrow$ & \\
\hline $\mathrm{P}$ & $\downarrow$ & & $\uparrow$ & \\
\hline Clav & $\downarrow$ & $\uparrow$ & & \\
\hline $\mathrm{Pl}$ & $\downarrow$ & & & $\uparrow$ \\
\hline Gong & $\downarrow$ & & & $\uparrow$ \\
\hline
\end{tabular}

Tabla 4. Entradas y salidas de instrumentos Evento D.

Fuente: Elaboración propia a partir del análisis de la instrumentación de Móvil II (Gramatges, 2001).

El corno funcionó como puente entre los eventos C y D y el compositor va reduciendo paulatinamente la cantidad de intérpretes.

\begin{tabular}{|c|c|c|}
\hline Inst. & & \\
\hline $\mathrm{P}$ (cordaje) & $\downarrow$ & $\uparrow$ \\
\hline P (teclado) & $\downarrow$ & $\uparrow$ \\
\hline Xil & $\downarrow$ & $\uparrow$ \\
\hline
\end{tabular}

Tabla 5. Entradas y salidas de instrumentos Evento E.

Fuente: Elaboración propia a partir del análisis de la instrumentación de Móvil II (Gramatges, 2001). 
RAFAEL GUZMÁN BARRIOS

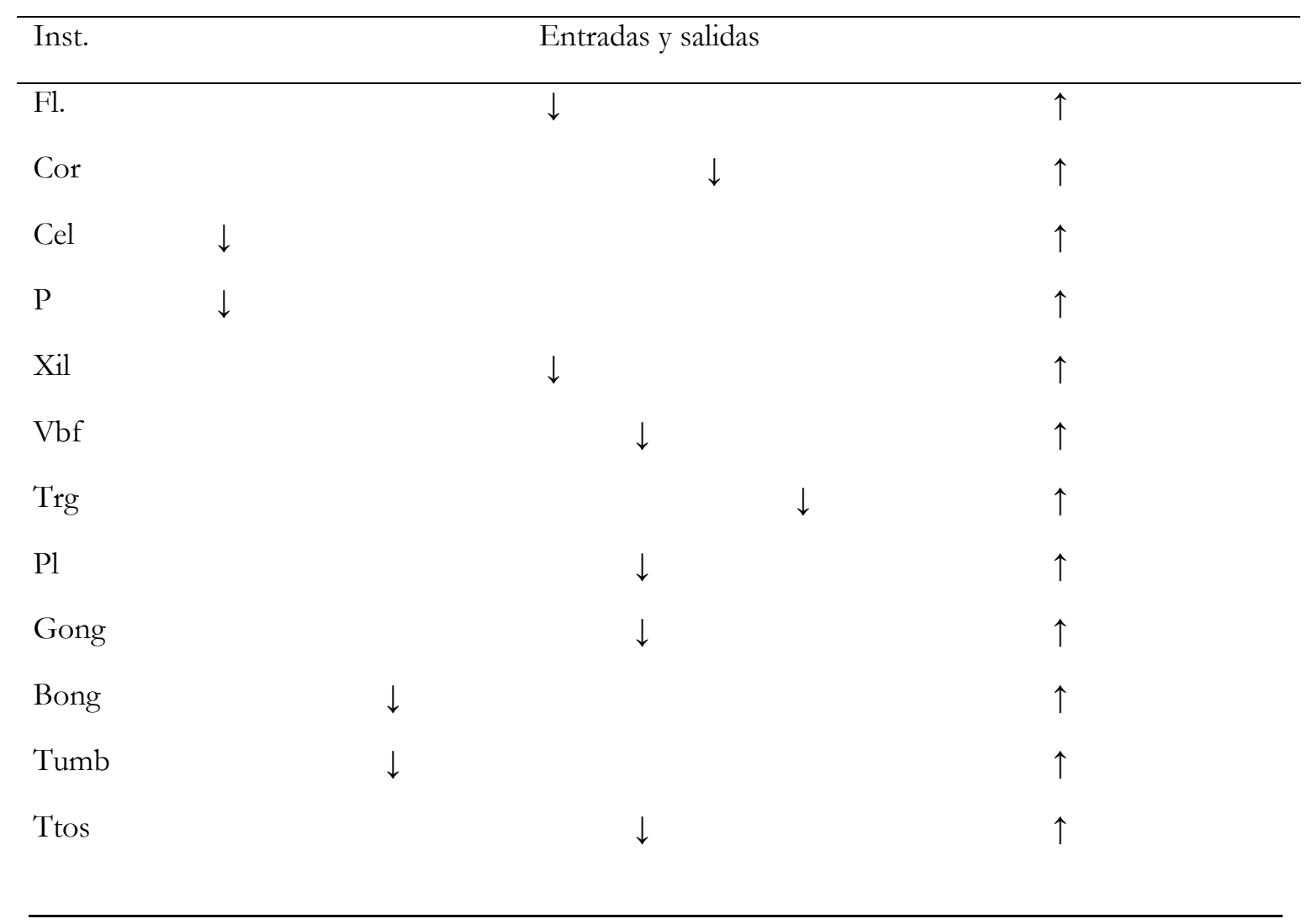

Tabla 6. Entradas y salidas de instrumentos Evento F.

Fuente: Elaboración propia a partir del análisis de la instrumentación de Móvil II (Gramatges, 2001).

El evento $\mathrm{F}$ tiene un alto grado de indeterminación pero el compositor no otorga al director ni a los intérpretes la libertad de las entradas y las retiradas. Contrastando con el evento anterior ahora participa casi todo el formato instrumental.

\begin{tabular}{lccc}
\hline Inst. & \multicolumn{3}{c}{ Entradas y salidas } \\
\hline Fl. & & $\downarrow$ & $\uparrow$ \\
Cor & & $\uparrow$ & \\
P & $\downarrow$ & & $\uparrow$ \\
Xil & & $\downarrow$ & $\uparrow$ \\
Vbf & & $\downarrow$ & $\uparrow$ \\
Marc & $\downarrow$ & & $\uparrow$ \\
Bong & $\downarrow$ & & $\uparrow$ \\
\hline
\end{tabular}

Tabla 7. Entradas y salidas de instrumentos Evento $G$.

Fuente: Elaboración propia a partir del análisis de la instrumentación de Móvil II (Gramatges, 2001). 
En los eventos F y $G$ los planos sonoros aparecen progresivamente y se retiran simultáneamente, enfatizando así la pausa entre las partes.

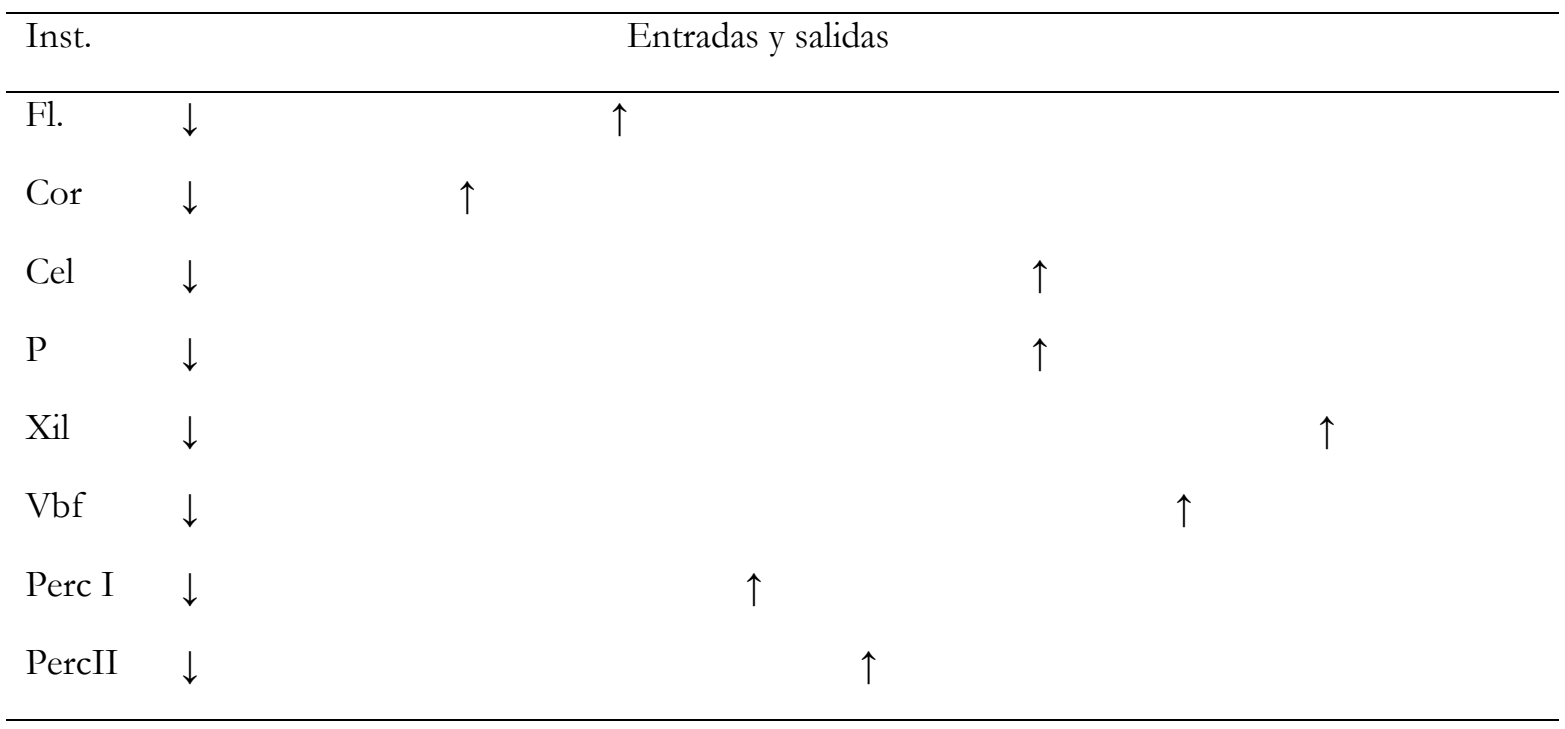

Tabla 8. Entradas y salidas de instrumentos Evento $H$.

Fuente: Elaboración propia a partir del análisis de la instrumentación de Móvil II (Gramatges, 2001).

\begin{tabular}{|c|c|c|c|c|c|c|c|}
\hline Inst. & & & & salidas & & & \\
\hline $\mathrm{Fl}$ & & & $\downarrow$ & $\uparrow$ & & & \\
\hline $\mathrm{P}$ & $\downarrow$ & & & & $\uparrow$ & & \\
\hline Vbf & & $\downarrow$ & & & & & $\uparrow$ \\
\hline Marc & & $\downarrow$ & & & & $\uparrow$ & \\
\hline
\end{tabular}

Tabla 9. Entradas y salidas de instrumentos Evento I.

Fuente: Elaboración propia a partir del análisis de la instrumentación de Móvil II (Gramatges, 2001).

\begin{tabular}{lccc}
\hline Inst. & & Entradas y salidas \\
\hline Corn & $\downarrow$ & $\uparrow$ & \\
Cel & $\downarrow$ & $\uparrow$ & \\
Clav & $\downarrow$ & $\uparrow$ & \\
Bong & $\downarrow$ & $\uparrow$ & \\
Marc & $\downarrow$ & & $\uparrow$ \\
\hline
\end{tabular}

Tabla 10. Entradas y salidas de instrumentos Evento J.

Fuente: Elaboración propia a partir del análisis de la instrumentación de Móvil II (Gramatges, 2001). 


\section{RAFAEL GUZMÁN BARRIOS}

Aunque en los eventos A, B, I, K y L las entradas y salidas también han sido progresivas, no existe simetría entre apariciones y retiradas de los planos sonoros. En estos eventos mencionados, excepto en $\mathrm{K}$, el último instrumento que se inserta es el primero que se retira, pero no se mantiene un orden regular general ya sea en espejo (simetría) o repitiendo en los finales el orden de las entradas.

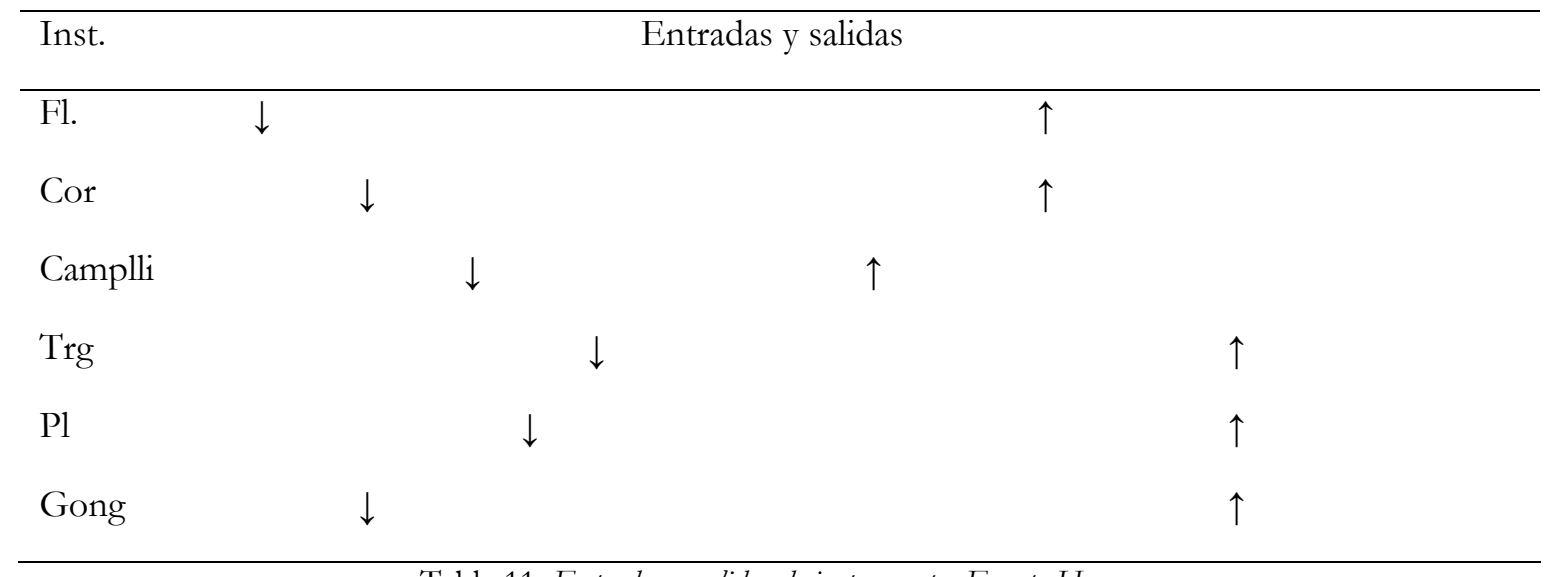

Tabla 11. Entradas y salidas de instrumentos Evento $H$.

Fuente: Elaboración propia a partir del análisis de la instrumentación de Móvil II (Gramatges, 2001).

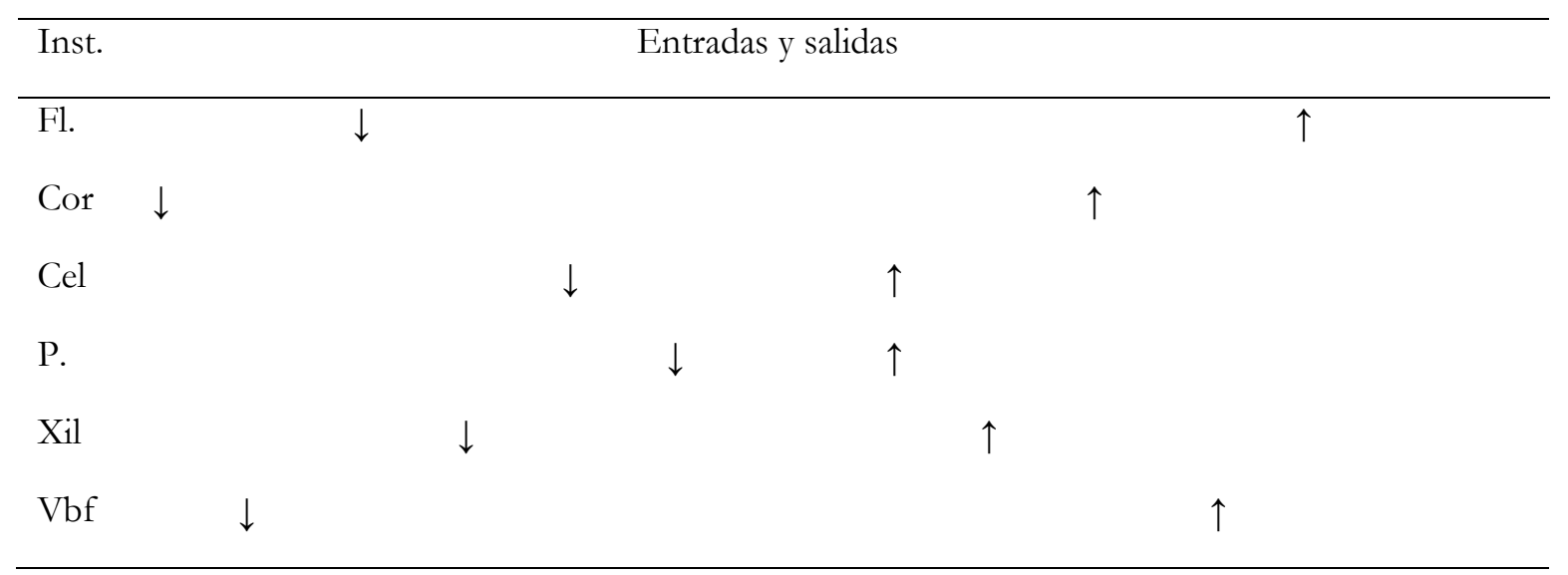

Tabla 12. Entradas y salidas de instrumentos Evento I.

Fuente: Elaboración propia a partir del análisis de la instrumentación de Móvil I (Gramatges, 2001).

La diversidad de formatos instrumentales que tienen los eventos, las múltiples variantes de entradas y salidas de los planos sonoros, el staff de baquetas a usar y el aprovechamiento que realiza el compositor a cada uno de los instrumentos, entre otros elementos, confirman que la riqueza tímbrica es la cualidad principal de la obra. 


\section{INTENSIDAD O DINÁMICA}

La dinámica o intensidad es uno de los elementos que distinguen a este Móvil del resto de la serie. Estamos ante una dinámica de línea con pendientes suaves, ya sea dentro de los eventos o entre ellos. Excepto en los eventos $\mathrm{F}$ y H, que la intensidad general la indica el compositor y la intensidad a nivel local la determina el intérprete, la dinámica no rebasa el $m f$ como promedio.

En la Figura 1 se muestra la gráfica donde el eje horizontal muestra los eventos en su orden de ejecución y el eje vertical refleja dos variables: la dinámica o intensidad, y el tiempo metronómico aproximado. Los colores representan la orquestación y también se traza la curva dramatúrgica de la obra, ya que los puntos de máxima intensidad coinciden con los eventos donde impera un alto grado de indeterminación, es decir, cuando se intensifican el conflicto estructuras-desplazamientos. Durante el resto de la obra predominan las sutilezas sonoras y las atmósferas acústicas inéditas. 
RAFAEL GUZMÁN BARRIOS

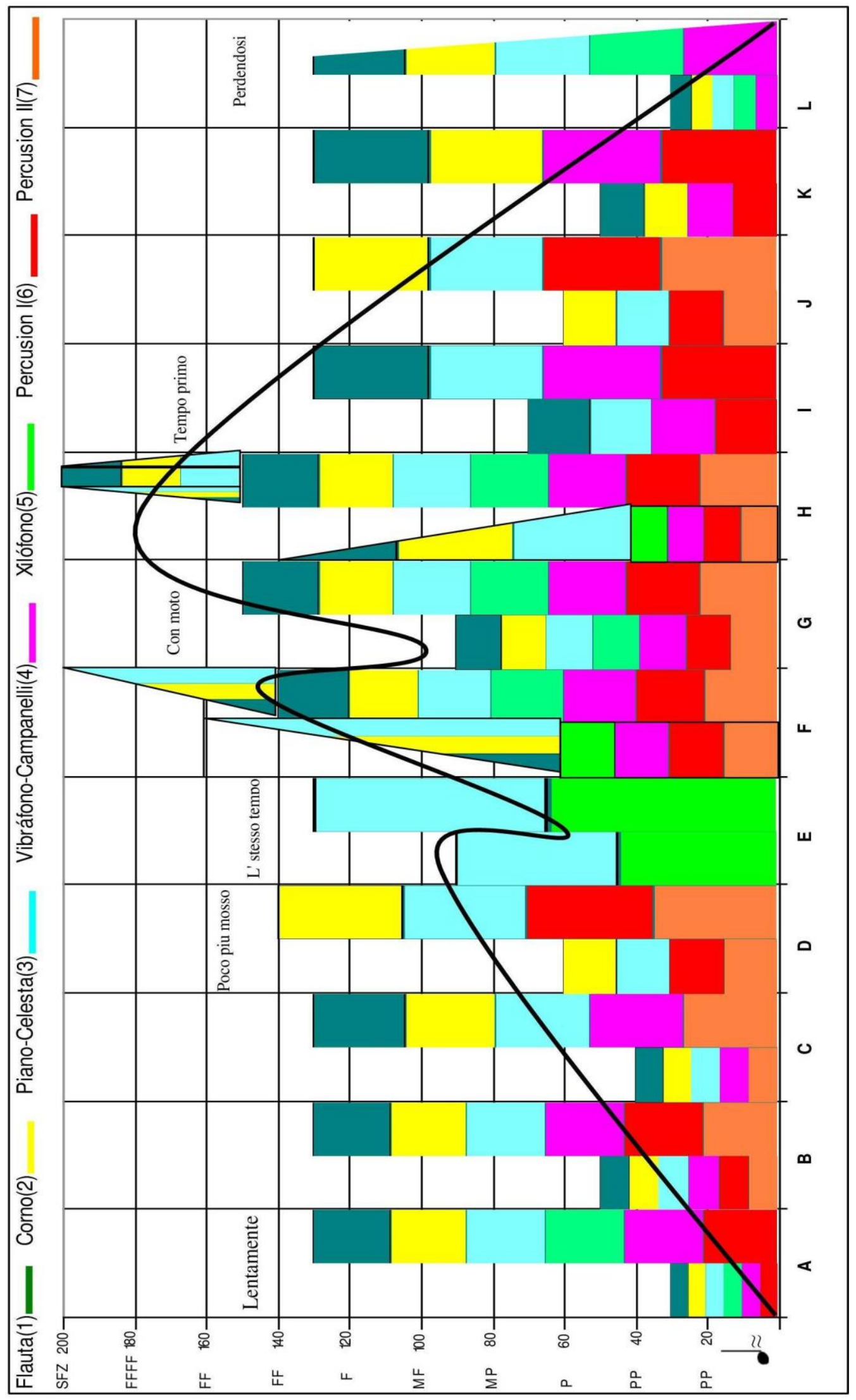

Fig. 1: Gráfico de tempo, intensidad y dramatúrgia Móvil I. 


\section{TEMPORALIDAD}

En este Móvil el director controla casi totalmente el carácter y la extensión de sus doce escenas. Acerca de la concepción del tiempo en la obra nos dice el compositor: "La obra no responde a una dimensión temporal estricta, el intérprete maneja lo que dura cada evento y fragmento" (H. Gramatges, comunicación personal, 21 de septiembre de 2005).

Dentro de cada evento, excepto los que tienen un alto grado de aleatoriedad, no existen ni bruscos ni frecuentes cambios dinámicos, temporales (a nivel general) y tímbricos; y finalizando casi todos ellos el compositor realiza una disminución gradual de la densidad instrumental (excepto en E, F, y G), por lo que esos cambios en el color del ensemble son muy sutiles y suaves. Las mutaciones dentro de los eventos son pocas por lo que la sensación del transcurrir del tiempo, aunque los eventos son cortos, es casi estática.

Casi todos los eventos mezclan tiempos lisos y estriados (Eventos A, B, C, G, I, K, L), otros manifiestan tiempos claramente estriados (Eventos D, E, J), y existen otros, como los eventos $\mathrm{F}$ y $\mathrm{H}$, que presentan un alto grado de indeterminación y su temporalidad está muy relacionada con el resultado final de ejecución.

\begin{tabular}{|c|c|c|c|c|c|c|}
\hline & $\mathrm{A}$ & B & $\mathrm{C}$ & I & $\mathrm{K}$ & $\mathrm{L}$ \\
\hline Liso & Vbf. y fl. & $\begin{array}{l}\text { Cordaje } \\
\text { P. y Gong }\end{array}$ & $\begin{array}{l}\text { Vbf. y } \\
\text { Perc II. }\end{array}$ & $\begin{array}{l}\text { Cordaje } \\
\text { P. y Vbf. }\end{array}$ & $\begin{array}{l}\text { Percusión } \\
\text { indeterminada }\end{array}$ & Vbf. y fl. \\
\hline Estriado & $\begin{array}{l}\text { P, Xil, } \\
\text { Cor y } \\
\text { Trg, }\end{array}$ & $\begin{array}{l}\text { Fl., Cor, } \\
\text { Camplli, } \\
\text { Marc y } \\
\text { Tumb. }\end{array}$ & $\begin{array}{l}\text { Fl., Cel } \\
\text { y P. }\end{array}$ & Fl. y Marc & $\begin{array}{l}\text { Flauta, corno y } \\
\text { glockenspiel }\end{array}$ & $\begin{array}{l}\text { P., Xil, Cor } \\
\text { y Cel. }\end{array}$ \\
\hline
\end{tabular}

Tabla 16. Eventos que mezclan tiempos lisos y estriados.

Fuente: Elaboración propia a partir del análisis del tiempo en el Móvil II (Gramatges, 2001).

La elección del tipo de baqueta a utilizar para cada instrumento tiene una estrecha relación con el resultado temporal y espacial de la obra. El frecuente uso de las escobillas, baquetas blandas o mangos, ante la poca utilización de las baquetas duras, añadido a la dinámica imperante (abundan los $p p$, $p$, $m p$ y $m f$ ), nos informa acerca de la atmósfera incierta y temporalmente intangible que desea el compositor.

En este Móvil el tiempo total percibido será una especie de superposición de los tiempos individuales que ha vivido cada intérprete. La experiencia temporal individual es ahora bien diferente porque cada parte tiene una subordinación estructural y no tonal con el resto. 


\section{RAFAEL GUZMÁN BARRIOS}

\section{ESPACIO MUSICAL}

El Móvil II está escrito para un conjunto de cámara cuya disposición espacial es determinada por el compositor sin posibilidad de movimiento alguno. Cada evento está concebido para un determinado y siempre diferente número de focos instrumentales, por lo que recibiremos cambios en las sensaciones de profundidad y relieve sonoro en cada sección de la obra. Podríamos decir que, aunque el formato general externo se mantiene inmóvil, internamente se garantiza al perceptor un inteligente movimiento espacial que cambia al unísono con la ocurrencia de los eventos. A continuación, mostramos la distribución y participación de los integrantes del ensemble en cada uno de los eventos. Los focos tachados no participan en la correspondiente sección.
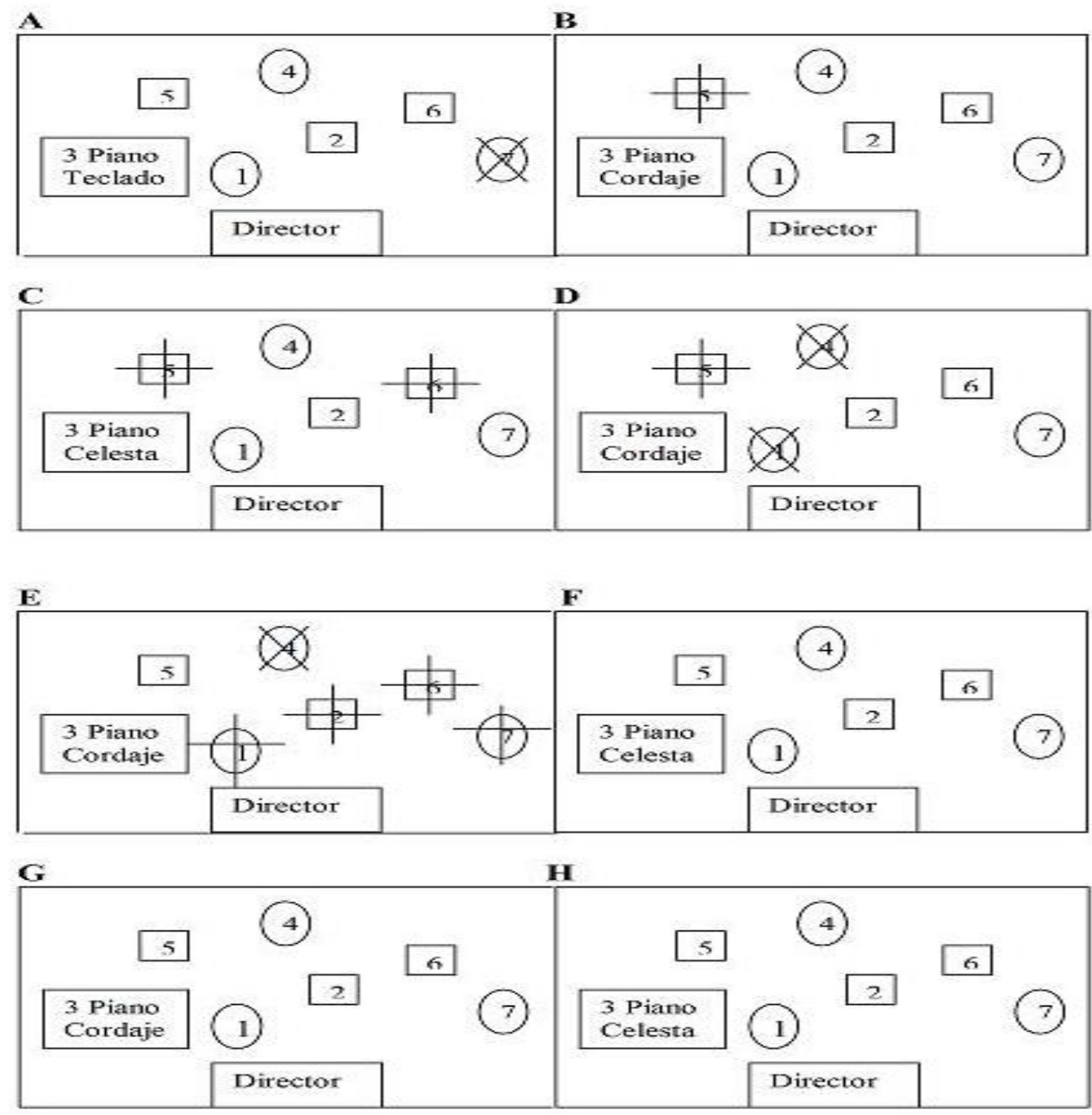

Fig. 2: Distribución espacial Móvil II. 1-Fl 2-Cor 3-P y Cel 4-Vbf y Camplli 5-Xil 6-Perc I 7-Perc II. 
Analizando las imágenes sobre la espacialidad externa en el Móvil II llegamos a las siguientes conclusiones:

- El compositor sitúa cerca del director a los instrumentos sonido más "débil" (piano y flauta) y así los va ubicando de acuerdo a su "potencia". Este criterio no será determinante en la obra debido a la forma en que Gramatges trabaja los medios sonoros y la dinámica.

- Todos los focos participan en los eventos F, G y H, pero en $G$ no intervienen: celesta, teclado del piano ni la percusión completa de cada grupo. Aunque en $\mathrm{H}$ se retoman los rectángulos indeterminados de $\mathrm{F}$, ambos tienen estructura morfológica, temporalidad y finales diferentes.

- Son casi iguales en la espacialidad externa los eventos D y J, pero en D el intérprete del foco 3 asume el cordaje del piano y en J la celesta. Las percusiones I y II son diferentes.

- Excepto en los eventos E y L, siempre está presente la percusión de sonido indeterminado.

Otro de los elementos que inciden en la espacialidad externomusical es la gestualidad escénica que en este caso el director del ensemble es el encargado de realizar los gestos adecuados para lograr la flexibilidad sonora que necesita la obra. El compositor declaró: "Manuel Duchesne Cuzán tuvo la cualidad de resolver plásticamente la obra" ( $\mathrm{H}$. Gramatges, comunicación personal, 21 de septiembre de 2005). 
RAFAEL GUZMÁN BARRIOS

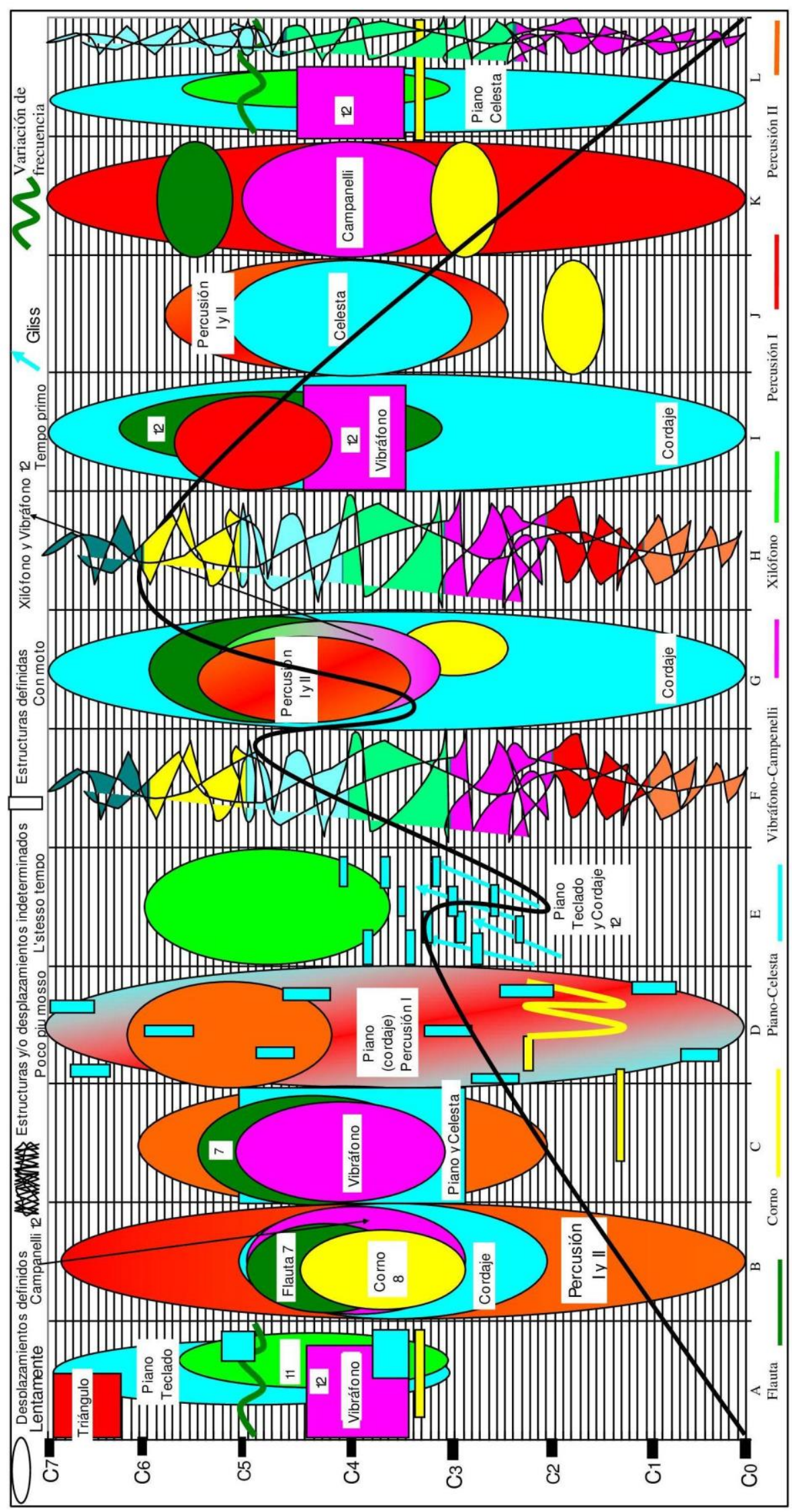

Fig. 3: Gráfico de espacialidad interna y dramatúrgia. 


\section{HAROLD GRAMATGES, ANÁLISIS MUSICOGRÁFICO DEL MóVIL II}

Acerca de la espacialidad interna en la obra el compositor nos dice:

En la obra existen momentos de mayor densidad y momentos de menor densidad. Se alternan los Tutti y los momentos de transparencia musical. Los instrumentos que utilizo como solista dialogan. Unas veces la flauta sola, otras el corno solo, y todo esto está rodeado por un mar que se mueve. (H. Gramatges, comunicación personal, 21 de septiembre de 2005).

El Gráfico 3 nos muestra el espacio intramusical del Móvil II, del cual podemos concluir:

- La obra aprovecha y utiliza en la mayoría de los eventos (excepto en A, E y J) todo o casi todo el espacio intramusical disponible.

-Es una obra eminentemente contrapuntística (considerando el término «contrapunto» desde la óptica de los planteamientos estéticos de las vanguardias musicales europeas como líneas o estratos sonoros que tienen una relación acústica-estructural y no tonal como en la tradición.) puesto que prevalecen los desplazamientos atonales.

- Son pocas las estructuras planteadas y generalmente lo hacen en forma de clusters.

- Existen otras manifestaciones sonoras como los microtonos (flauta) y los glissandis (cordaje del piano).

\section{Dramaturgia}

Existen dos pares dialécticos fundamentales que rigen la línea dramática en esta obra: Indeterminación-Determinación (el tiempo es el único elemento que es maleable e incierto, el resto de los elementos, alturas e intensidades, pueden ser precisados o no por el compositor); y en un nivel más localizado nos encontramos con el conflicto Estructurasdesplazamientos.

Ya desde el evento A comienzan los conflictos entre los desplazamientos, las estructuras y los microtonos que plantea la flauta. El tiempo es flexible para todos, pero lo es más para corno, flauta y vibráfono, que para el resto. En B, aunque no existen estructuras, aumenta el grado de indeterminación que se manifiesta en piano (cordaje) y la percusión I y II. Los eventos C y D manifiestan un claro incremento de los dos conflictos fundamentales que caracterizan esta obra, hasta que en $\mathrm{E}$ los intérpretes se encuentran los desplazamientos y las estructuras bien precisados por el compositor; esta disminución del grado de indeterminación se verá rápidamente alterada por una repentina improvisación general (evento F) que provoca la evaporación de las diferencias entre estructuras y desplazamientos, y el predominio abrumador de la indeterminación. 


\section{RAFAEL GUZMÁN BARRIOS}

Los eventos F, G y H establecen las crestas en la línea dramática porque todo el ensemble reflejará la agudización de los conflictos Indeterminación-Determinación y Estructuras-Desplazamientos de forma desequilibrada.

$\mathrm{F} \rightarrow$ Alto grado de indeterminación. El intérprete define las estructuras y los desplazamientos.

$\mathrm{G} \rightarrow$ Excepto en el tiempo, el resto de los elementos están bien precisados en el corpus musical. Predominan los desplazamientos.

$\mathrm{H} \rightarrow$ Tiene las mismas características que $\mathrm{F}$.

Durante los cuatro eventos finales se retorna a las condiciones de equilibrio para los pares en conflicto. Disminuye la densidad del ensemble, predominio de los desplazamientos en J y K, mayor grado de indeterminación en I y L.

\section{ANÁLISIS GENERAL}

El aumento de la densidad instrumental está relacionado con las crestas en la curva dramatúrgica. En las pendientes, a los lados de las cúspides, se prioriza el cromatismo tímbrico y la sutileza del sonido. Aunque la curva dramatúrgica presenta semejanzas con el comportamiento de la dinámica, consideramos este hecho como casual puesto que son los conflictos indeterminación-determinación y estructuras-desplazamientos los que mueven la narrativa de la obra y no la intensidad. Suponiendo que los eventos F, G y H estuvieran bajo una dinámica de $\not p p$, la línea dramática no sufriría cambio alguno.

Los trazos horizontales predominan ampliamente sobre el pensamiento vertical. Las estructuras utilizadas generalmente están en función del color y la atmósfera deseada (eventos $\mathrm{C}$ y E) y no como pensamiento armónico tonal o atonal. En ocasiones el tejido se torna tan tupido e indescifrable (eventos $\mathrm{F}$ y $\mathrm{H}$ ), que sería mejor hablar de una diagonalidad en la textura.

El timbre es indiscutible protagonista de esta obra. La mayoría de los eventos muestran una búsqueda sutil de colores mediante mezclas siempre cambiantes de los participantes y a través del inteligente empleo de la instrumentación (baquetas) y la dinámica. $\mathrm{Ni}$ siquiera en el evento final se re-expone con el mismo formato; el compositor recalca ideas con paletas sonoras diferentes. Sin embargo, en los eventos $\mathrm{F}$ y $\mathrm{H}$ es el caos, aunque siempre hay un resultado tímbrico determinado, el que se apodera de las riendas de la obra.

La obra plantea un conflicto a nivel global entre la Indeterminación real y la indeterminación aparente. Esta última es el efecto de indeterminación que recibe el perceptor, y sin embargo la mayoría de los parámetros musicales están bien precisados por el compositor. La temporalidad flexible e intangible es la piedra angular y el elemento fundamental que garantiza esta continua experiencia aleatoria. 


\section{CONCLUSIONES}

El Móvil II de Harold Gramatges responde al pensamiento de experimentación y ruptura propio de la década del 60, porque se incorpora plenamente a la corriente transgresora predominante en esos años que sustituyó los viejos conceptos (melodía, armonía, polifonía) por otros de diferente naturaleza (microtonalismo, aleatoriedad parcial, espacialidad, nueva temporalidad).

Dos pares dialécticos fundamentales garantizan el desarrollo y puesta en marcha de la obra analizada: determinación e indeterminación y estructuras-desplazamientos, aunque respecto a este último par, son los desplazamientos los verdaderos protagonistas. El atonalismo libre empleado en la obra tiene sus raíces en la música atonal y dodecafónica europea, pero no acude al serialismo rígido de los compositores vanguardistas de la segunda posguerra. Las incursiones microtonales, el empleo de clusters, el uso de bloques y masas sonoras como conglomerados geométricos en continuo movimiento a lo largo y ancho del espectro audible, así como la constante alternancia de condensaciones y rarefacciones de sonidos hacen de esta obra compactas construcciones donde se manifiesta en todos sus frentes el espacio intramusical.

El azar y lo aleatorio son herramientas que el compositor utiliza controladamente: las duraciones e indicaciones metronómicas aproximadas y las señales de entrada para el director, confirman que en el pareja de contrarios (determinación-indeterminación) radica la esencia del pensamiento estético-musical de Harold Gramatges aplicado al Móvil II. La temporalidad flexible y subjetiva junto con el tratamiento de la espacialidad, son la columna vertebral de esta obra. Las frecuentes apariciones de indeterminados accelerandos y ritardandos, la ausencia de líneas divisorias e indicaciones de valores aproximados en el tempo son algunos elementos que garantizan un mundo temporal rico, flexible y susceptible de infinitas lecturas.

Si la percepción de un tiempo suspendido consiste en reducir las mutaciones para hacerlas imperceptibles, entonces en el Móvil II cada evento es un presente eterno. Hay una tendencia al tiempo estático en cada parte; encontramos en el objeto musical la fractura entre los eventos y la continuidad sin apenas contrastes dentro de cada uno de ellos; el compositor acude a la filosofía temporal meditativa del lejano Oriente.

La obra analizada constituye una marcada ruptura con el pasado neoclásico del compositor en la década de los 50, aunque esta incursión dentro de los nuevos procedimientos técnicos no alcanza los extremismos de la indeterminación total al estilo del norteamericano John Cage, ni el serialismo integral a la manera de Pierre Boulez. A pesar del grado de indeterminación presente en la obra, es el compositor quien proporciona la identidad a la inmensa mayoría de los acontecimientos musicales.

Respecto a la presencia o no de lo cubano en el Móvil $I I$, esta obra tiene abundante percusión cubana pero el compositor no tiene la intención de sugerir una evidente cubanía. $\mathrm{Al}$ respecto nos dice: "La percusión no hace ninguna célula rítmica de nuestros toques de clave sino crear una atmósfera que tímbricamente se reconozca como nuestra... no existen 


\section{RAFAEL GUZMÁN BARRIOS}

citas rítmicas específicas, debe crear un ambiente peculiar que ubique al perceptor en las Antillas” (H. Gramatges, comunicación personal, 21 de septiembre de 2005).

El Móvil II constituye un regreso a nuestras más ancestrales raíces. El corno con sus efectos de apertura y cierre del pabellón, los sonidos insistentes e imprevisibles temporalmente, y la flauta con sus incursiones microtonales hacen de esta obra una evocación a lo que fuimos, un viaje sonoro a la semilla. "En esta obra veo a los indios caribeños, uno tocando la flauta, otro el corno y lo demás es el ambiente que les rodea: el agua y demás cosas añadidas”. (H. Gramatges, comunicación personal, 21 de septiembre de 2005). Una cubanidad diferente evoca el Móvil II: cubanía precolombina.

Se ha analizado solo una parte del corpus, es decir una de las piezas de la serie Móviles. Y el análisis de una muestra generalmente nos ayuda a comprender la totalidad del segmento (Nattiez, 2005, p.12) En ese sentido el Móvil II es una obra estilísticamente representativa de la totalidad del corpus, en este caso no solo la serie de 4 piezas, sino también de la obra del compositor en los años 60 .

\section{BIBLIOGRAFÍA}

Acosta, L. (2001). Los Móviles de Harold Gramatges [libro de partituras]. La Habana: Ed. Letras Cubanas.

Boulez, P. (2009). Pensar la música hoy. Murcia: Colegio Oficial de Aparejadores y Arquitectos Técnicos de la Región de Murcia, Colección de Arquilectura 52.

Gramatges, H. (2001). Móviles [ed. facsimilar]. La Habana: Ed. Letras Cubanas.

Guzmán, R. (2019). La Muerte del Guerrillero: análisis músico-gráfico. Índex. Revista de arte contemporáneo, (7), pp. 58-66.

López Cano, R. (2001). Del Cronos al Topos. Espacio y tiempo en las músicas del siglo XX.

En M. Vega Rodríguez \& C. Villar Taboada (Coords.). El tiempo en las músicas del siglo XX (pp. 101-122). Valladolid: Ed. Glares Gestión Cultural.

Nattiez, J. J. (2005) La semiología musical: más allá del estructuralismo, después del postmodernismo... Boletin Música, 15-16, pp. 3-56. La Habana: Casa de las Américas.

Padilla, A. (1999). El Análisis musical dialéctico. (Tesis Doctoral). Helsinki: Universidad de Helsinki.

Pérez, S. (2011). Las Diferentes Acepciones de Forma y Estructura en la Historia del Análisis Musical. Tiempo y Sociedad, (5), pp. 89-98. 


\section{HAROld GRAMATGES, ANÁlisis MUSICOGRÁfICO DEL MóVIL II}

Rodríguez, M. (2006) Eclecticism in modern cuban music as reflected in selected piano works by Harold Gramatges: an investigative analysis. (Tesis Doctoral). Lincoln: University of Nebraska.

Sánchez, J. A. (2010). Dramaturgia en el campo expandido. Repensar la dramaturgia, errancia y transformación. Murcia: Centro Párraga. Centro de Documentación y Estudios avanzados de Arte Contemporáneo.

Vela, M. (2018). Análisis gráfico-sonoro como fuente de conocimiento de la obra musical en estudiantes de la Universidad Internacional de La Rioja (UNIR). REDU. Revista de docencia universitaria, 16(2), pp. 231-247.

Fecha de recepción: 18/02/2020

Fecha de aceptación: 23/09/2020 